\title{
Neuroprotective role of dexmedetomidine pretreatment in cerebral ischemia injury via ADRA2A-mediated phosphorylation of ERK1/2 in adult rats
}

\author{
YANYAN SHI ${ }^{1}$, XIAO-HONG PENG ${ }^{1}$, XIA LI $^{1}$, GAO-PING LUO ${ }^{1}$ and MING-FU WU ${ }^{2}$ \\ ${ }^{1}$ Department of Anesthesia, Wuhan Fourth Hospital, Puai Hospital, Tongji Medical College, \\ Huazhong University of Science and Technology, Wuhan, Hubei 430033; ${ }^{2}$ Cancer Biology Research Center, \\ Tongji Hospital, Tongji Medical College, Huazhong University of Science and Technology, Wuhan, Hubei 430030, P.R. China
}

Received April 18, 2018; Accepted September 27, 2018

DOI: $10.3892 /$ etm.2018.6878

\begin{abstract}
Neuroprotective effects of dexmedetomidine (Dex) have been reported in various models of brain injury. However, to our knowledge, the neuroprotective mechanism of Dex pretreatment in rats remains unknown. The aim of the present study was to detect the expression of the $\alpha 2 \mathrm{~A}$ adrenergic receptor (ADRA2A) in focal ischemic brain tissues and to investigate the protective role and corresponding mechanism of Dex pretreatment in cerebral ischemia in rats. A hypoxia/reoxygenation $(\mathrm{H} / \mathrm{R})$ cell model in primary cultured astrocytes and a focal cerebral ischemia/reperfusion (I/R) model in adult rats were used. The expression of ADRA2A and extracellular signal-regulated kinases 1 and 2 (ERK1/2) in the primary cultured astrocytes and rat brain ischemic tissues was detected in the different conditions prior to and following
\end{abstract}

Correspondence to: Dr Ming-Fu Wu, Cancer Biology Research Center, Tongji Hospital, Tongji Medical College, Huazhong University of Science and Technology, 1095 Jiefang Road, Wuhan, Hubei 430030, P.R. China

E-mail: mingfuwutj@163.com

Abbreviations: Dex, dexmedetomidine; I/R, ischemia/reperfusion; GFAP, glial fibrillary acidic protein; ADRA2A, $\alpha 2 \mathrm{~A}$ adrenergic receptor; ERK1/2, extracellular-signal regulated kinases 1 and 2; $\mathrm{H} / \mathrm{R}$, hypoxia/reoxygenation; (t)MCAO, (transient) middle cerebral artery occlusion; GDNF, glial cell line-derived neurotrophic factor; SD, Sprague-Dawley; BCA, bicinchoninic acid; p-, phosphorylated; EGFR, epidermal growth factor receptor; TTC, triphenyl tetrazolium chloride; d-cAMP, dibutyryl cyclic-adenosine monophosphate; CCA carotid artery; ECA, external carotid artery; ICA, internal carotid artery; CNS, central nervous system; EGF, epidermal growth factor; EGFR, epidermal growth factor receptor; HB-EGF, heparin-binding epidermal growth factor-like growth factor

Key words: astrocytes, extracellular-signal regulated kinases 1 and 2, $\alpha 2 \mathrm{~A}$ adrenergic receptor, dexmedetomidine, cerebral ischemia/reperfusion injury
Dex pretreatment using western blotting. The H/R model of primary cultured astrocytes and the focal cerebral I/R model in adult rats were successfully constructed. Under the normal oxygen conditions, $500 \mathrm{ng} / \mathrm{ml}$ Dex pretreatment increased the expression of ADRA2A and phosphorylated (p)-ERK1/2 in the astrocytes compared with in the control group. Hypoxic culture for $6 \mathrm{~h}$ and then reoxygenation for $24 \mathrm{~h}$ decreased the levels of p-ERK1/2 in the astrocytes compared with those in control group. This decrease was prevented by Dex pretreatment for $3 \mathrm{~h}$. The hypoxic culture and then reoxygenation increased the expression of ADRA2A. Similarly, compared with those prior to Dex treatment, the levels of ADRA2A and p-ERK1/2 in the brain ischemic tissues following Dex treatment were increased. The levels of ADRA2A and p-ERK1/2 were $0.72 \pm 0.23$ and $0.66 \pm 0.25$ following Dex treatment, compared with $0.76 \pm 0.22$ and $0.31 \pm 0.18$, respectively, prior to Dex treatment. The effect of Dex pretreatment increasing p-ERK1/2 expression was attenuated by AG1478 pretreatment. In summary, Dex appeared to promote phosphorylation of ERK1/2 in astrocytes under H/R. As a specific agonist of ADRA2A, Dex may activate phosphorylation of ERK1/2 via ADRA2A in astrocytes. Thus, the neuroprotective role of Dex pretreatment against cerebral ischemic injury may function via ADRA2A-mediated phosphorylation of ERK1/2.

\section{Introduction}

Cerebral ischemia/reperfusion (I/R) injury is a key and common pathological process in certain diseases of the nervous system, including stroke and traumatic brain injury (TBI), and is also among the main causes of disability and mortality $(1,2)$. Although clinical trials have been conducted in an attempt to treat $\mathrm{I} / \mathrm{R}$ injury-related diseases, the only efficacious methods for the treatment of stroke and TBI are thrombolysis and hypothermia $(1,2)$. Therefore, it is of great importance to elucidate the pathophysiological mechanism of cerebral I/R and to develop an effective neuroprotective intervention strategy such as brain protectants. The clinical failure of many potential neuroprotective strategies involves a lack of understanding with regards to the choice of treatment windows for defined targets, the importance of neurons interacting 
with astrocytes and, in particular, the newly identified role of astrocytes, including the expression of neurotransmitter receptors and the defense against oxidative stress $(1,3)$. Literature on the mechanism of cerebral I/R injury is mainly focused on oxidative stress, excitatory neurotoxicity and $\mathrm{Ca}^{2+}$ overload (2). However, the activation of astrocytes serves a vital role in neurodegenerative changes, including cerebral ischemia and hippocampal neuron damage by tumor necrosis factor- $\alpha$-mediated inflammatory injury $(1,3,4)$. Astrocytes are activated and proliferate in order to promote repair of neurons, axon growth and nerve function restoration in the early stage of cerebral ischemia (5).

Dexmedetomidine (Dex) is a selective $\alpha 2$ adrenoceptor agonist that is used as a potent sedative for critically ill patients in the intensive care unit and as an effective anesthetic adjuvant for surgical patients in the operating room (6). Dex has been demonstrated to improve neuronal survival following transient global or focal cerebral ischemia in rats $(7,8)$, which provides a novel neuroprotective target for cerebral ischemia, but researchers hold different views on the brain protective mechanism of Dex. Previous studies have explained the neuroprotection of Dex in an $\alpha 2$ adrenoceptor-dependent manner. Kose et al (9) reported that intravenous drug delivery of Dex served an important protective role in cerebral ischemia-mediated neuron injury by inhibiting the activation of astrocytes. Meanwhile, others have observed that reduction of glutamic acid agonist-induced neuronal apoptosis by Dex was associated with increased expression of brain-derived neurotrophic factor in astrocytes (10). These results indicate that the neuroprotective effect of Dex pretreatment is associated with the function and $\alpha 2$ adrenoceptor expression of astrocytes during cerebral I/R injury.

The neuroprotective effect of Dex has been demonstrated to involve extracellular signal-regulated kinases 1 and 2 (ERK1/2) in an $\alpha 2$ adrenoceptor-independent manner. For instance, Dex may stimulate glial cell line-derived neurotrophic factor (GDNF) release in order to rescue neurons from neurotoxicity induced by oxygen-glucose deprivation by upregulating hippocampal ERK1/2 expression, and the effects may be attenuated by inhibition of ERK $(11,12)$. However, during cerebral I/R injury, whether $\alpha 2 \mathrm{~A}$ adrenergic receptor (ADRA2A)-mediated phosphorylation of ERK1/2 is involved in the neural protection of Dex remains unknown to the best of our knowledge.

In the present study, a hypoxia/reoxygenation $(\mathrm{H} / \mathrm{R})$ model of primary cultured astrocytes and a focal cerebral I/R model in adult rats were used to investigate the effect of Dex pretreatment on the expression of ADRA2A and phosphorylation of ERK1/2 in astrocytes during hypoxic culture and cerebral ischemic conditions, respectively. The neuroprotective mechanism of Dex pretreatment was demonstrated to be caused by ADRA2A-mediated phosphorylation of ERK1/2.

\section{Materials and methods}

Animals. The present study used 24 2-day-old neonatal male Sprague-Dawley (SD) rats weighing 250-280 g, obtained from the Experimental Animal Center of Tongji Medical College of Huazhong University of Science and Technology (HUST), Wuhan, China. The rats were individually fed with standard pellet chow and had access to drinking water ad libitum under standard conditions $\left(22-24^{\circ} \mathrm{C}, 40-70 \%\right.$ humidity and a regular 12-h day/night cycle). The Ethics Committee for the Use of Experimental Animals at Tongji Medical College of HUST approved all experimental procedures.

Chemicals. Dex was manufactured and supplied by Jiangsu Hengrui Medicine Co., Ltd. (Jiangsu, China). Rat glial fibrillary acidic protein (GFAP; cat. no. P90068Hu01) and recombinant rat ADRA2A monoclonal antibodies (cat. no. CSB-YP001388MO) were purchased from Shanghai Yanhui Biotechnology Co., Ltd. (Shanghai, China) and Wuhan Huamei Biotechnology Co., Ltd. (Beijing, China) and used for immunofluorescent staining and protein blotting during the following experiments. Goat anti-rabbit (cat. no. BA1055) or goat anti-rat (cat. no. BA1058) horseradish peroxidase-conjugated secondary antibodies and a bicinchoninic acid (BCA) protein assay kit were purchased from Wuhan Boster Biological Technology Ltd. (Wuhan, China). Anti- $\beta$-actin rabbit monoclonal antibody (cat. no. 4970), rabbit ERK1/2 (cat. no. 4348) and phosphorylated (p)-ERK1/2 (Thr202/Tyr204; cat. no. 9101) antibodies, and the epidermal growth factor receptor (EGFR) tyrosine kinase inhibitor AG1478 were obtained from Cell Signaling Technology, Inc. (Danvers, MA, USA). Triphenyl tetrazolium chloride (TTC) was acquired from Sigma-Aldrich (Merck KGaA, Darmstadt, Germany).

Primary cortical astrocyte culture. The protocol for the culture of primary cortical astrocytes was performed according to an improved method of that first proposed by McCarthy (13). Briefly, the cerebral cortical tissues of the neonatal SD rats were detached and segmented, digested for $30 \mathrm{~min}$ in $2.5 \mathrm{~g} / 1$ trypsin at $37^{\circ} \mathrm{C}$, and mixed with Dulbecco's modified Eagle's medium (Sigma-Aldrich; Merck KGaA) containing 15\% heat-inactivated fetal bovine serum (Sigma-Aldrich; Merck $\mathrm{KGaA}$ ) to end the digestion. The digested cerebral cortices were centrifuged at $560 \mathrm{xg}$ for $5 \mathrm{~min}$ and the resuspended cells were filtered with a mesh bag $(200 \mathrm{~mm})$ in order to achieve a single cell suspension. The prepared single cell suspension was inoculated into a glass culture bottle without polylysine to remove the fibroblasts for $15 \mathrm{~min}$ at $37^{\circ} \mathrm{C}$ and $5 \% \mathrm{CO}_{2}$, and then the cell suspension in the flask was gently flipped and plated onto a 50-ml glass culture bottle coated with L-polylysine at a density of $3 \times 10^{6}$ cells/bottle. After 8-12 days of plating, monolayers of astrocytes were obtained. The growth state of astrocytes was observed under a light microscope (magnification, x100). The adherent cells were digested for $2-3 \mathrm{~min}$ in $300 \mu \mathrm{l}$ of $2.5 \mathrm{~g} / 1$ trypsin at $37^{\circ} \mathrm{C}$. Immunofluorescent staining for GFAP was used to assess the purity of the primary cortical astrocytes. Astrocytes were cultured on cover glasses $(18 \times 18 \mathrm{~mm})$ placed in 6-well plates at $37^{\circ} \mathrm{C}$ until the cells attained a confluence of $70-80 \%$. Cells were fixed using $4 \%$ formaldehyde at room temperature for $30 \mathrm{~min}$ and then incubated at $37^{\circ} \mathrm{C}$ for $30 \mathrm{~min}$ in $0.01 \mathrm{~mol} / \mathrm{l} \mathrm{PBS}$ containing $10 \%$ goat serum (Wuhan Boster Biological Technology Ltd.) and $0.1 \%$ Triton X-100. Following the addition of rat GFAP $\left(1: 100\right.$; cat. no. P90068Hu01) at $4^{\circ} \mathrm{C}$ overnight and washing three times with $0.01 \mathrm{~mol} / \mathrm{l} \mathrm{PBS}, \mathrm{Cy} 3$ labeled sheep anti-rat $\operatorname{IgG}$ (1:100; cat. no. A0507; Beyotime Institute of Biotechnology, Haimen, China) was added and 
incubated free from light at $37^{\circ} \mathrm{C}$ for $30 \mathrm{~min}$. The slides with the cells were sealed using $30 \mu \mathrm{l}$ mounting medium containing DAPI at room temperature for $30 \mathrm{~min}$. The stained sections were examined with a Leica fluorescence microscope, and images were captured with a CCD camera (14). Dibutyryl cyclic-adenosine monophosphate (d-cAMP; $150 \mu \mathrm{mol}$, Sigma, St. Louis, MO, USA) was added to induce cell maturation over a duration of 3 to 4 days at $37^{\circ} \mathrm{C}$ when $>95 \%$ of the cultured cells were GFAP positive.

$H / R$ cell model and experimental protocol. In vitro experimental ischemia was induced by $\mathrm{H} / \mathrm{R}$ using an anoxic incubation method (15). The primary astrocytes cultured in glucose-free medium were transferred from the normal incubator containing $22 \% \mathrm{O}_{2}$ and $5 \% \mathrm{CO}_{2}$ at $37^{\circ} \mathrm{C}$ to an anaerobic chamber containing $94 \% \mathrm{~N}_{2}, 1 \% \mathrm{O}_{2}$ and $5 \% \mathrm{CO}_{2}$ at $37^{\circ} \mathrm{C}$ for $6 \mathrm{~h}$, and then the glucose-free medium was replaced with Dulbecco's modified Eagle medium containing $10 \%$ heat-inactivated fetal bovine serum (Sigma-Aldrich; Merck $\mathrm{KGaA}$ ) and $1 \%$ penicillin/streptomycin and cells were returned to the normal incubator for reoxygenation for $24 \mathrm{~h}$. In the preliminary experiment, cells seeded in 6-well plates prior to Dex treatment were used to investigate the effect of Dex pretreatment on the primary astrocytes in hypoxic conditions. The primary in-vitro cultured astrocytes were divided into 5 groups and were subjected to various treatments as follows: i) Control (Con)+Dex 2, cells were treated with $500 \mathrm{ng} / \mathrm{ml} \mathrm{Dex}$ for $3 \mathrm{~h}$ and then incubated in the normal incubator for $27 \mathrm{~h}$; ii) Con, cells were incubated in the normal incubator for $30 \mathrm{~h}$; iii) $\mathrm{H} / \mathrm{R}$, cells were incubated according to the conditions of the $\mathrm{H} / \mathrm{R}$ model; iv) $\mathrm{H} / \mathrm{R}+\mathrm{Dex} 1$, cells were pretreated with $100 \mathrm{ng} / \mathrm{ml}$ Dex for $3 \mathrm{~h}$ and then incubated according to the conditions of the $\mathrm{H} / \mathrm{R}$ model; and v) $\mathrm{H} / \mathrm{R}+\mathrm{Dex} 2$, cells were pretreated with $500 \mathrm{ng} / \mathrm{ml}$ Dex for $3 \mathrm{~h}$ and then incubated according to the conditions of the $\mathrm{H} / \mathrm{R}$ model.

Construction of the focal cerebral I/R model and drug administration. The cerebral I/R model was induced by transient middle cerebral artery occlusion (tMCAO) according to a previous study (16). Briefly, the right carotid artery (CCA), the external carotid artery (ECA) and the internal carotid artery (ICA) were carefully isolated under sterile conditions following rat anesthesia with $3 \%$ isoflurane. Following temporary closure of the ICA and CCA with arteriole clips, a silicone line (6-0; Doccol Corporation, Sharon, MA, USA) was inserted from the right CCA into the ICA to occlude the origin of the right middle cerebral artery. After $30 \mathrm{~min}$ of occlusion, the silicone line was withdrawn, the arteriole clip was lessened and the ECA was ligated permanently to allow reperfusion. Following surgery, the rats were separately fed water and food ad libitum at room temperature. As displayed in Fig. 1, the rats were randomly divided into 4 groups ( $n=6$ rats/group) as follows: i) Sham, the same surgical procedures without tMCAO were performed and the rats were intravenously infused with $\mathrm{DMSO}$ and $0.9 \% \mathrm{NaCl}$ at the same volume as that in the Dex-pretreated rats; ii) I/R, the rats endured ischemia for $90 \mathrm{~min}$ and reperfusion for $24 \mathrm{~h}$; iii) Dex-pretreated (Dex $+\mathrm{I} / \mathrm{R})$, Dex (bolus, $3 \mu \mathrm{g} / \mathrm{kg}$ ) was infused through the left femoral vein at the onset of ischemia and then infusion continued for a further $2 \mathrm{~h}(6 \mu \mathrm{g} / \mathrm{kg} / \mathrm{h})$; and iv) $\mathrm{AG}+\mathrm{Dex}+\mathrm{I} / \mathrm{R}$, rats were injected intracerebroventricularly with $1 \mu \mathrm{M}$ AG1478 prior to the operation, Dex pretreatment and reperfusion (17). The changes of physiological variables including $\mathrm{pCO}_{2}, \mathrm{pO}_{2}, \mathrm{MAP}$, $\mathrm{pH}$ and blood glucose at different I/R periods, such as at the onset of ischemia, $30 \mathrm{~min}$ of ischemia and $24 \mathrm{~h}$ of reperfusion in ischemic rats were observed and recorded.

Evaluation of neurological function and cerebral infarct volume. A five-point scoring method was adopted to assess the extent of neurological deficit in the rats $(8,18)$. Briefly, the motor findings were scored as follows: 0 points, asymptomatic symptoms; 1 point, forelimb flexion; 2 points, forelimb flexion and decreased resistance to lateral push; 3 points, forelimb flexion, decreased resistance to lateral push and unilateral circling; and 4 points, forelimb flexion, and unable or difficult to ambulate. Following decapitation, the brain tissue of each rat was immediately collected and frozen at $-20^{\circ} \mathrm{C}$ for $30 \mathrm{~min}$. The frozen brain was cut into $4-\mathrm{mm}$ thick slices and the slices were stained in $2 \%$ TTC solution $(\mathrm{pH} 7.4)$ at $37^{\circ} \mathrm{C}$ for $30 \mathrm{~min}$ and fixed in $10 \%$ formalin at room temperature for $20 \mathrm{~min}$. Normal brain tissue stained red and the infarcted area white. The volume of cerebral infarction was measured and calculated using Image-Pro Plus software, version 6.0 (Media Cybernetics, Inc., Rockville, MD, USA). Infarct volume $(\%)=($ left cerebral hemispheric volume-right cerebral non-infarct volume)/(left cerebral hemisphere volume $\mathrm{x} 2$ ) x100 (19).

Hematoxylin and eosin $(H \& E)$ staining. Following immersion of rat brain tissues in $4 \%$ paraformaldehyde solution for $24 \mathrm{~h}$ at $4^{\circ} \mathrm{C}$, the water in the brain tissue was gradually removed with 70, 80, 90 and $95 \%$ ethanol solutions, which was followed by xylene clearing and paraffin embedding. The embedded wax blocks were sectioned into $5-\mu \mathrm{m}$ continuous coronal brain tissue slices, dried and dewaxed, and then were stained with H\&E at room temperature for $30 \mathrm{~min}$ (Solarbio Co., Ltd, Beijing, China). Morphological characteristics of each brain tissue section were observed with an optical microscope (Leica DM1000; Leica Microsystems GmbH, Wetzlar, Germany).

Western blotting. Total protein from the rat right ischemic hemisphere or primary cultured astrocytes were extracted on ice with cell lysis buffer [1\% Triton X-100, $50 \mathrm{mM}$ Tris (pH 7.4), $150 \mathrm{mM} \mathrm{NaCl}, 0.1 \%$ sodium dodecyl sulfate (SDS) and $1 \mathrm{mM}$ EDTA] containing $1 \mathrm{mmol} / 1$ complete proteinase inhibitor cocktail. The BCA method was used to determined protein concentration. Total protein $(40-50 \mathrm{mg}$ ) was separated by $10 \%$ SDS-polyacrylamide gel electrophoresis and then electrotransferred onto nitrocellulose membranes. The membranes were blocked with $5 \%$ non-fat powdered milk and then incubated overnight at $4{ }^{\circ} \mathrm{C}$ with the antibodies against rabbit ERK1/2 $(1: 1,000)$ and p-ERK1/2 $(1: 1,000)$, rat ADRA2A $(1: 1,000)$ and rabbit $\beta$-actin $(1: 1,000)$. Subsequently, the membranes were washed in tris-buffered saline with Tween-20, goat anti-rabbit or goat anti-rat secondary antibodies were added and the color was developed using an enhanced chemiluminescence kit (cat. no. orb90504; Wuhan Boster Biological Technology Ltd.). The values were assayed using Quantity One software (version 4.62, Bio-Rad Laboratories, Inc., Hercules, CA, USA). The relative optical density of $\mathrm{p}$-ERK1/2/ERK1/2 was 


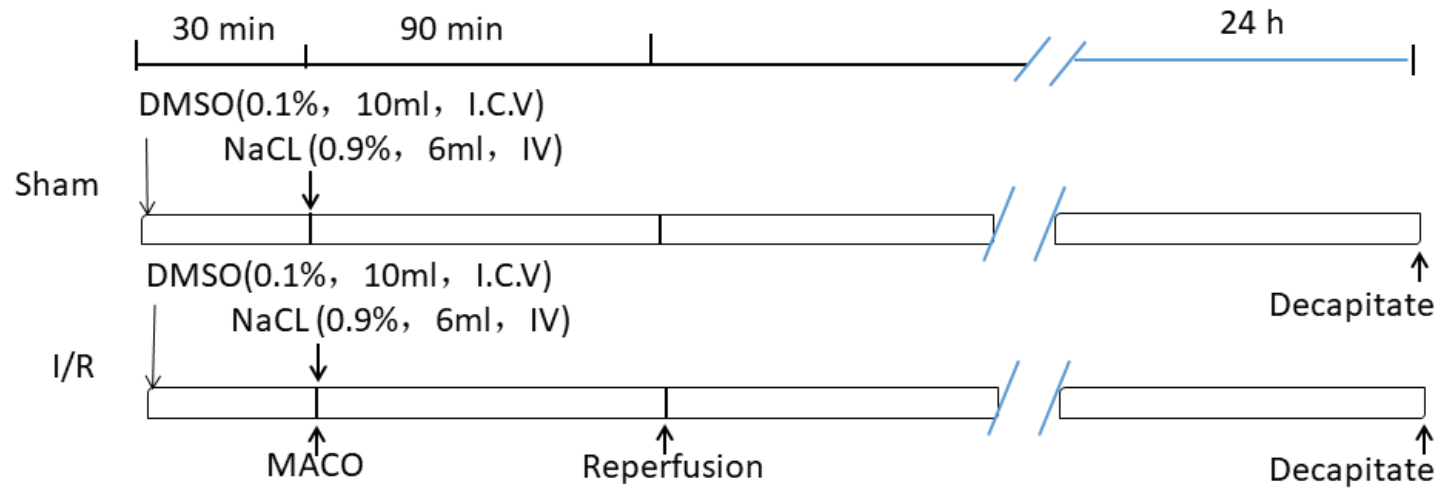

$\operatorname{DMSO}(0.1 \%, 10 \mathrm{ml}$, I.C.V)
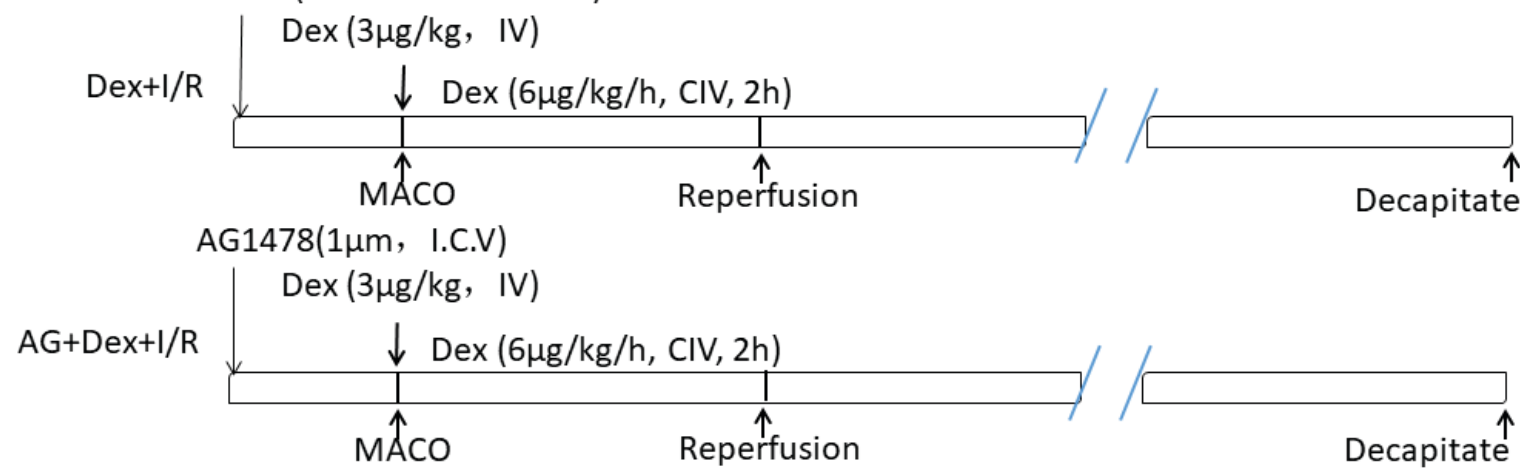

Figure 1. Experimental groups and drug delivery protocols for brain protection by Dex treatment in ischemia/reperfusion rat models.

calculated as the phosphorylation level of ERK1/2. The relative optical density of ADRA2A/ $\beta$-actin was calculated as the expression level of ADRA2A.

Statistical analysis. SPSS statistical software version 19.0 (IBM Corp., Armonk, NY, USA) was used for data analysis. All quantitative data are expressed as the mean \pm standard deviation. One-way analysis of variance followed by the Newman-Keuls test was used to make statistical comparisons. $\mathrm{P}<0.05$ was considered to indicate a statistically significant difference.

\section{Results}

Morphological changes and GFAP expression in the primary cultured astrocytes. To investigate the role of astrocytes in cerebral ischemic injury, primary cultured astrocytes from the cerebral cortex of newborn SD rats were separated, cultivated and purified through an improved McCarthy method combined with trypsin digestion, differential adhesion and vibration on a thermostatic table. The astrocytes with a purity of $98 \%$ and which exhibited typical layers of growth were collected and cultured. The adherent cells covered the bottom of the bottle and exhibited clear growth in a stratified manner under a light microscope after 9 to 12 days culture (Fig. 2A). The immunofluorescence results indicated that there were numerous astrocytes expressing GFAP to a high level in the primary culture after 12 days. When cells grew to fuse, the cell protrusions were not clear. They were diverse in shape and size and the cell protrusions were elongated at the edges of cells (Fig. 2B).
Effect of DEX pretreatment on ADRA2A and ERK1/2 expression in primary cultured astrocytes under $H / R$ conditions. Astrocytes have important roles in normal and pathological functioning of the central nervous system (CNS). A previous study reported that ADRA2A colocalizes with GFAP in primary astrocytes as identified by a double immunofluorescence assay (14). To observe the neuroprotective role and corresponding mechanism of DEX pretreatment in cerebral I/R injury involving ADRA2A in vitro in the present study, an astrocyte $\mathrm{H} / \mathrm{R}$ model was constructed.

The levels of ERK1/2 expression in the astrocytes were not notably different among the groups except for those in the control group. Under the normal oxygen conditions, $500 \mathrm{ng} / \mathrm{ml}$ Dex pretreatment increased the expression levels of ADRA2A and $\mathrm{p}-\mathrm{ERK} 1 / 2$ in the astrocytes, compared with those in the control group $(\mathrm{P}<0.01)$. Hypoxic culture for $6 \mathrm{~h}$ and then reoxygenation for $24 \mathrm{~h}$ decreased the levels of p-ERK1/2 expression in the astrocytes compared with those in the control group $(\mathrm{P}<0.01)$, and this was prevented by Dex pretreatment (100 or $500 \mathrm{ng} / \mathrm{ml}$ ) for $3 \mathrm{~h}$ in the H/R + Dex groups, in which p-ERK1/2 expression was increased $(\mathrm{P}<0.01)$. The hypoxic culture and then reoxygenation increased the expression levels of ADRA2A ( $\mathrm{P}<0.01$; Fig. 3). These results suggested that Dex promotes phosphorylation of ERK1/2 in astrocytes under H/R conditions. As a specific agonist of ADRA2A, Dex may activate phosphorylation of ERK1/2 through ADRA2A in astrocytes.

Successful construction of the focal cerebral ischemia model. A focal cerebral I/R model was constructed to investigate the neuroprotective role of Dex pretreatment in cerebral ischemic injury. The regular variables were detected during 

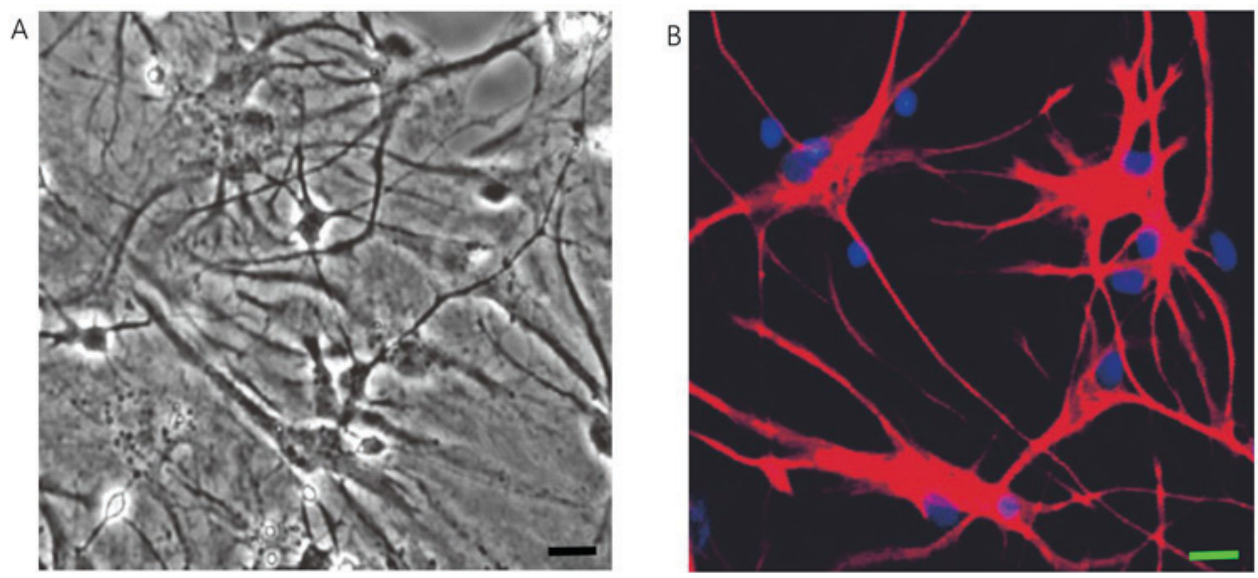

Figure 2. Morphological changes and GFAP expression in primary cultured astrocytes. (A) The morphology of astrocytes under an inverted phase contrast microscope after 12 days of culture. Scale bar, $50 \mu \mathrm{m}$. Magnification, x200. (B) Double-labeling immunofluorescence of GFAP (red) and DAPI (blue) in primary cultured astrocytes at day 7. Scale bar, $25 \mu \mathrm{m}$. Magnification, x400. GFAP, glial fibrillary acidic protein.

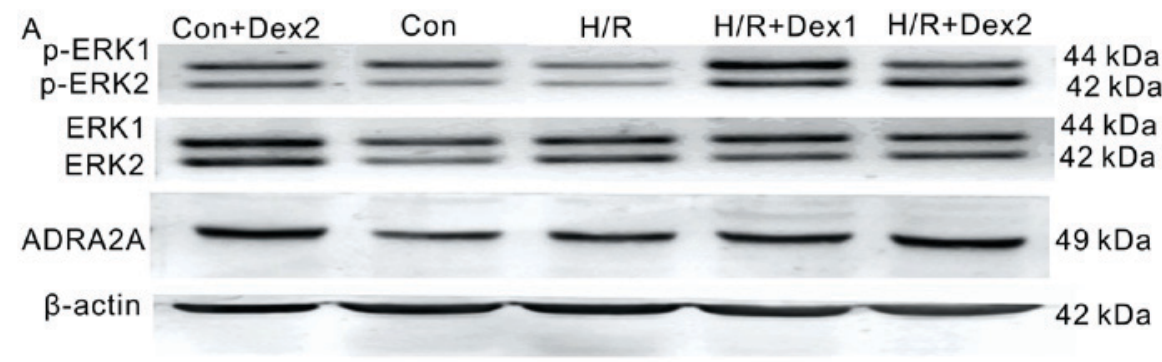

B

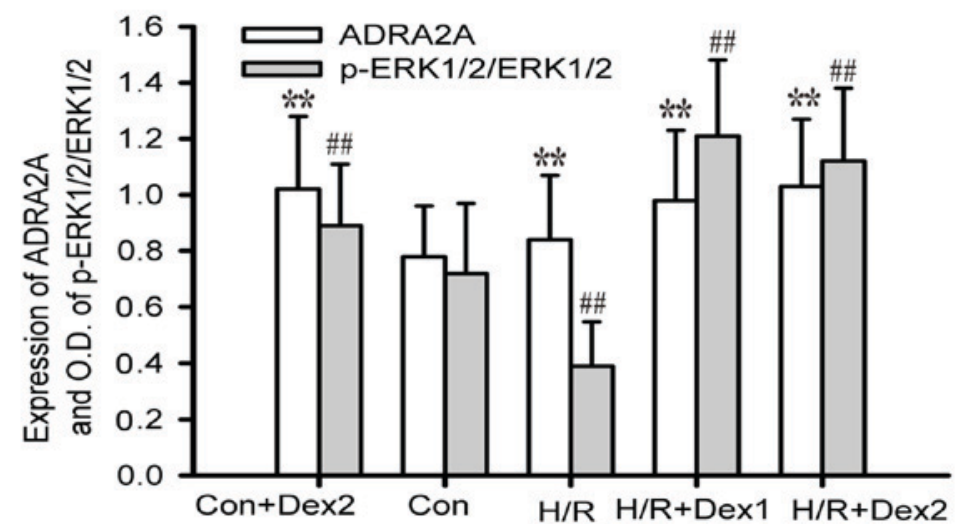

Figure 3. Effect of Dex pretreatment on ADRA2A and ERK1/2 expression in primary cultured astrocyte under H/R conditions. (A) Expression of ADRA2A and ERK1/2 as detected by western blotting after different treatment conditions. (B) Quantification of the western blot data for ADRA2A and ERK1/2 expression after different treatment conditions. The relative optical density of p-ERK1/2/ERK1/2 was calculated, and the expression of ADRA2A and p-ERK1/2/ERK1/2 was normalized. Dex was administered $3 \mathrm{~h}$ before H/R. Data are expressed as the mean \pm standard deviation. ${ }^{* *} \mathrm{P}<0.01$ and ${ }^{\# \#} \mathrm{P}<0.01 \mathrm{vs}$. control group. Dex, dexmedetomidine; ADRA2A, $\alpha 2 \mathrm{~A}$ adrenergic receptor; ERK, extracellular signal-regulated kinase; H/R, hypoxia/reoxygenation; p-, phosphorylated.

the surgical procedure. There were no notable differences in the baseline physiological values such as $\mathrm{pCO}_{2}, \mathrm{pO}_{2}$ and $\mathrm{pH}$ between the I/R and I/R Dex groups (Table I). Compared to those following $30 \mathrm{~min}$ of ischemia and $2 \mathrm{~h}$ of reperfusion in the I/R group, the levels of MAP were significantly reduced and then returned to normal, while blood glucose levels were markedly increased and then returned to normal in the Dex $+\mathrm{I} / \mathrm{R}$ group $(\mathrm{P}<0.01)$. These results indicated that the focal cerebral ischemia model was successful and suitable for Dex pretreatment protection of brain function when subject to cerebral ischemic injury.
Dex pretreatment increases neural protection in focal cerebral $I / R$ rat models. The neurological deficit score and cerebral infarct volume in the I/R group were higher than those in the sham-operated group $(\mathrm{P}<0.01$; Fig. $4 \mathrm{~A})$. Dex pretreatment notably reduced the damage to neurological function $(\mathrm{P}<0.01$; Fig. 4B) and brain infarct volume induced by $\mathrm{I} / \mathrm{R}(\mathrm{P}<0.01$; Fig. 4C and D).

Dex pretreatment upregulates ADRA2A and ERK1/2 expression in focal cerebral I/R brain tissues. The expression levels of ADRA2A in the ischemic brain tissues of the I/R group were 
Table I. Change of physiological variables at different $\mathrm{I} / \mathrm{R}$ periods in ischemic rats.

\begin{tabular}{|c|c|c|c|c|c|c|}
\hline \multirow[b]{2}{*}{ Variable } & \multicolumn{2}{|c|}{ Baseline } & \multicolumn{2}{|c|}{$30 \mathrm{~min}$ of ischemia } & \multicolumn{2}{|c|}{$2 \mathrm{~h}$ of reperfusion } \\
\hline & $\mathrm{I} / \mathrm{R}$ & $\operatorname{Dex}+\mathrm{I} / \mathrm{R}$ & $\mathrm{I} / \mathrm{R}$ & $\operatorname{Dex}+\mathrm{I} / \mathrm{R}$ & $\mathrm{I} / \mathrm{R}$ & $\operatorname{Dex}+\mathrm{I} / \mathrm{R}$ \\
\hline $\mathrm{pH}$ & $7.23 \pm 0.01$ & $7.25 \pm 0.02$ & $7.24 \pm 0.02$ & $7.23 \pm 0.03$ & $7.22 \pm 0.03$ & $7.23 \pm 0.01$ \\
\hline MAP (mmHg) & $94 \pm 6$ & $93 \pm 7$ & $108 \pm 10$ & $87 \pm 6^{\mathrm{a}}$ & $96 \pm 9$ & $95 \pm 8$ \\
\hline $\mathrm{pCO}_{2}(\mathrm{mmHg})$ & $41 \pm 1$ & $40 \pm 2$ & $42 \pm 1$ & $43 \pm 2$ & $40 \pm 2$ & $43 \pm 2$ \\
\hline $\mathrm{pO}_{2}(\mathrm{mmHg})$ & $93 \pm 6$ & $94 \pm 5$ & $90 \pm 2$ & $89 \pm 3$ & $92 \pm 6$ & $92 \pm 7$ \\
\hline Blood glucose (mM/l) & $5.9 \pm 0.7$ & $6.6 \pm 0.6$ & $5.0 \pm 0.7$ & $9.5 \pm 0.7^{\mathrm{a}}$ & $5.3 \pm 0.5$ & $6.2 \pm 0.5$ \\
\hline
\end{tabular}

${ }^{\mathrm{a}} \mathrm{P}<0.05, \mathrm{I} / \mathrm{R}$ vs. Dex+I/R. I/R, ischemia/reperfusion; Dex, dexmedetomidine; MAP, mean arterial pressure; $\mathrm{pCO}_{2} / \mathrm{O}_{2}$, partial pressure of carbon dioxide/oxygen.

higher than those of the sham group, while p-ERK1/2/ERK expression was higher in the Sham group than in the I/R group. These effects were prevented by Dex pretreatment. The expression levels of ADRA2A and p-ERK1/2 were $0.72 \pm 0.23$ and $0.66 \pm 0.25$ following Dex treatment, respectively, compared with $0.76 \pm 0.22$ and $0.31 \pm 0.18$, respectively, prior to Dex treatment. The effect of Dex pretreatment on increasing p-ERK1/2 expression was attenuated by AG1478 pretreatment in the ischemic brain tissues (Fig. 5A and B). These results indicated that the neuroprotective role of Dex pretreatment in cerebral ischemic injury functions may occur via ADRA2A-mediated phosphorylation of ERK1/2.

\section{Discussion}

Recent in-vitro experimental studies have indicated that Dex pretreatment against cerebral ischemic damage in cultured brain slices from the hippocampus induced neuroprotection via ERK1/2 phosphorylation $(20,21)$. In the present study, Dex promoted phosphorylation of ERK1/2 in astrocytes under $\mathrm{H} / \mathrm{R}$ conditions. As a specific agonist of ADRA2A, Dex may activate phosphorylation of ERK1/2 through ADRA2A in astrocytes. Thus, the neuroprotective role of Dex pretreatment against cerebral ischemia injury may function via ADRA2A-mediated phosphorylation of ERK1/2.

As astrocytes are the most abundant subtype of cells in the CNS, they are structurally and functionally involved in normal brain function and responses to an ischemic lesion $(22,23)$. A number of studies have indicated that astrocytes are better preserved than neurons in animal models of stroke outside of the core in which all cells die, and that astrocytes are important therapeutic targets for improving functional outcome, including after stroke $(1-4,22)$. The many essential functions of astrocytes include $\mathrm{K}^{+}$homeostasis, neurotransmitter synthesis and uptake, synapse formation and regulation of the blood brain barrier $(3,23)$. GFAP expression occurs during reperfusion periods and increases in the peri-infarct area (24). In the present study, GFAP expression was preserved following MCAO-induced focal cerebral I/R, which indicates that astrocytes serve key roles in ischemic brain diseases.

An increasing number of studies have indicated that astrocytes have important supporting and regulatory roles in brain function (1-3). The essential metabolic needs of neurons include $\mathrm{K}^{+}$buffering, glutamate clearance, brain antioxidant defense and the modulation of neuronal excitability (23). The astrocytic contributions to brain injury are extremely complex and not completely understood, but it is established that astrocytes cause brain injury-associated neuronal death (22). A previous study reported that failure of astrocytes to support these needs of neurons leads to this secondary injury (22).

Which of the three subtypes of $\alpha 2$ adrenoceptor, namely $\alpha 2 \mathrm{~A}, \alpha 2 \mathrm{~B}$ and $\alpha 2 \mathrm{C}$, mediates the neuroprotective effect of Dex was examined in cell culture and in an in vivo model of neonatal asphyxia (25). Although the $\alpha 2 \mathrm{~A}$ and $\alpha 2 \mathrm{C}$ subtypes are dominant in the CNS, all three subtypes are widely distributed in the nervous system (25). The different functions of the receptor subtypes result from their specific distributions in brain tissues. In the mammalian CNS, the $\alpha 2 \mathrm{~B}$ receptor subtype is mainly located in the thalamus, the a2A subtype is highly expressed in the locus coeruleus (in the brain stem), and the $\alpha 2 \mathrm{~A}$ and $\alpha 2 \mathrm{C}$ subtypes are widely distributed in the brain (25). ADRA2A also exists in the presynaptic and postsynaptic terminals, where it is mainly involved with the inhibition of norepinephrine release and the excitement of neurons $(26,27)$. Dex mediates its main pharmacological and cranial nerve protective effects via ADRA2A (28). In vivo activation of the locus coeruleus may produce $\alpha 2 \mathrm{~A}$ adrenergic effects in astrocytes, while in the $\mathrm{CNS}$, the target cell type of $\alpha 2 \mathrm{~A}$ adrenergic agonists is the astrocytes (28). Notably, astrocytes treated with d-cAMP mainly express ADRA2A (29). Compared with neurons, endothelial cells and microglia, adult mouse astrocytes have been demonstrated to express ADRA2A more highly in the cerebral cortex (30); thus, the key site of the effect of Dex in the whole brain appears to be astrocytes. It was previously reported that ADRA2A was expressed and colocalized with GFAP in astrocytes (14). In the present study, there were numerous astrocytes highly expressing GFAP in primary culture; however, a similar experiment to confirm that GFAP was expressed together with ADRA2A was not performed and should be verified in future study. In vitro studies have further demonstrated that in cultured astrocytes and brain slices, Dex activation of ADRA2A is dependent on the concentration of Dex $(8,29)$. Although doses of 1-100 $\mu \mathrm{g} / \mathrm{kg}$ Dex may have a neuroprotective effect during ischemia (31), it is not clear if Dex pretreatment prior to focal cerebral 

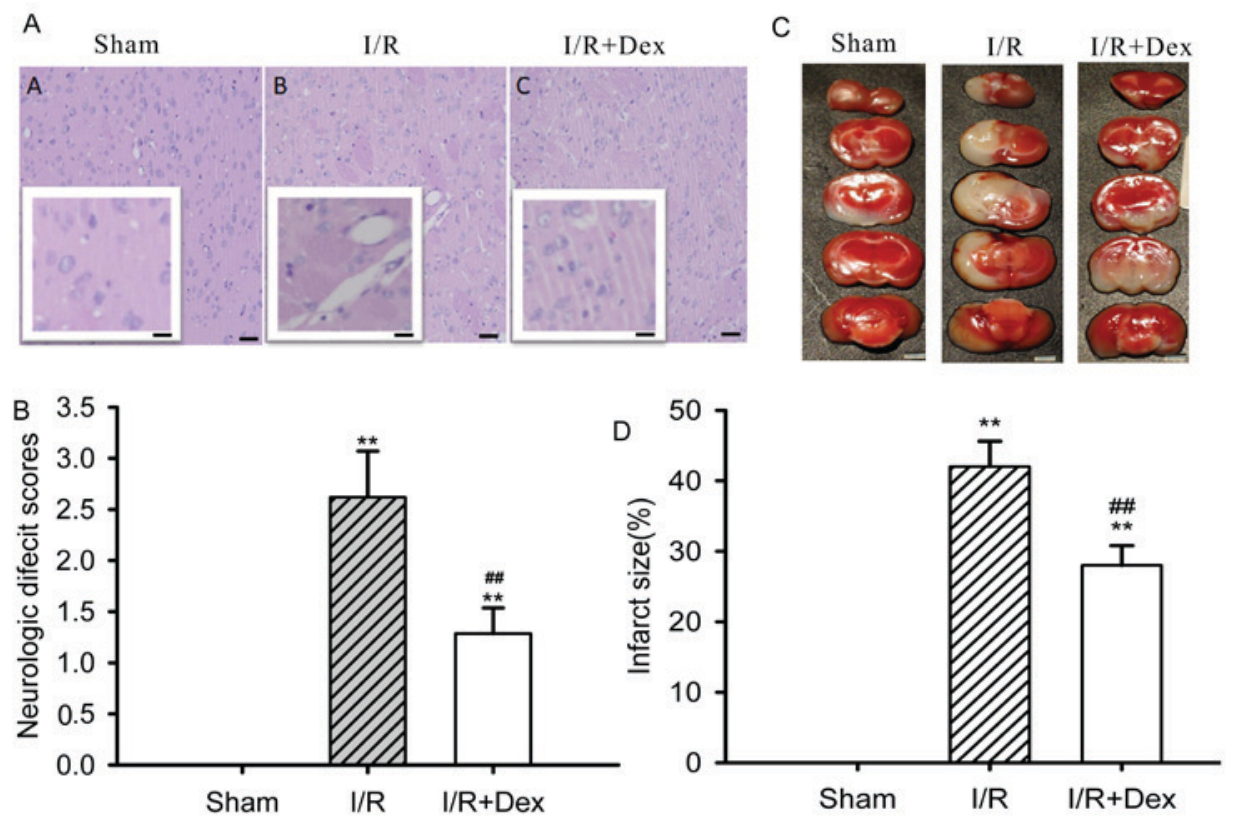

Figure 4. Dex pretreatment increases neural protection in a focal cerebral I/R rat model. (A) Hematoxylin and eosin staining of the brain tissues. In the main microscope image, scale bar, $50 \mu \mathrm{m}$. Magnification, x200; in the enlarged section, scale bar, $25 \mu \mathrm{m}$. Magnification, x400. (B) Neurological deficit scores following Dex pretreatment. (C, D) Cerebral infarct volume in I/R rat models subject to middle cerebral artery occlusion. (C) Triphenyl tetrazolium chloride staining of representative sections. (D) Quantification of infarct volume. ${ }^{* *} \mathrm{P}<0.01$ vs. sham group; ${ }^{\# \#} \mathrm{P}<0.01 \mathrm{vs}$. I/R group. I/R, ischemia/reperfusion; Dex, dexmedetomidine.
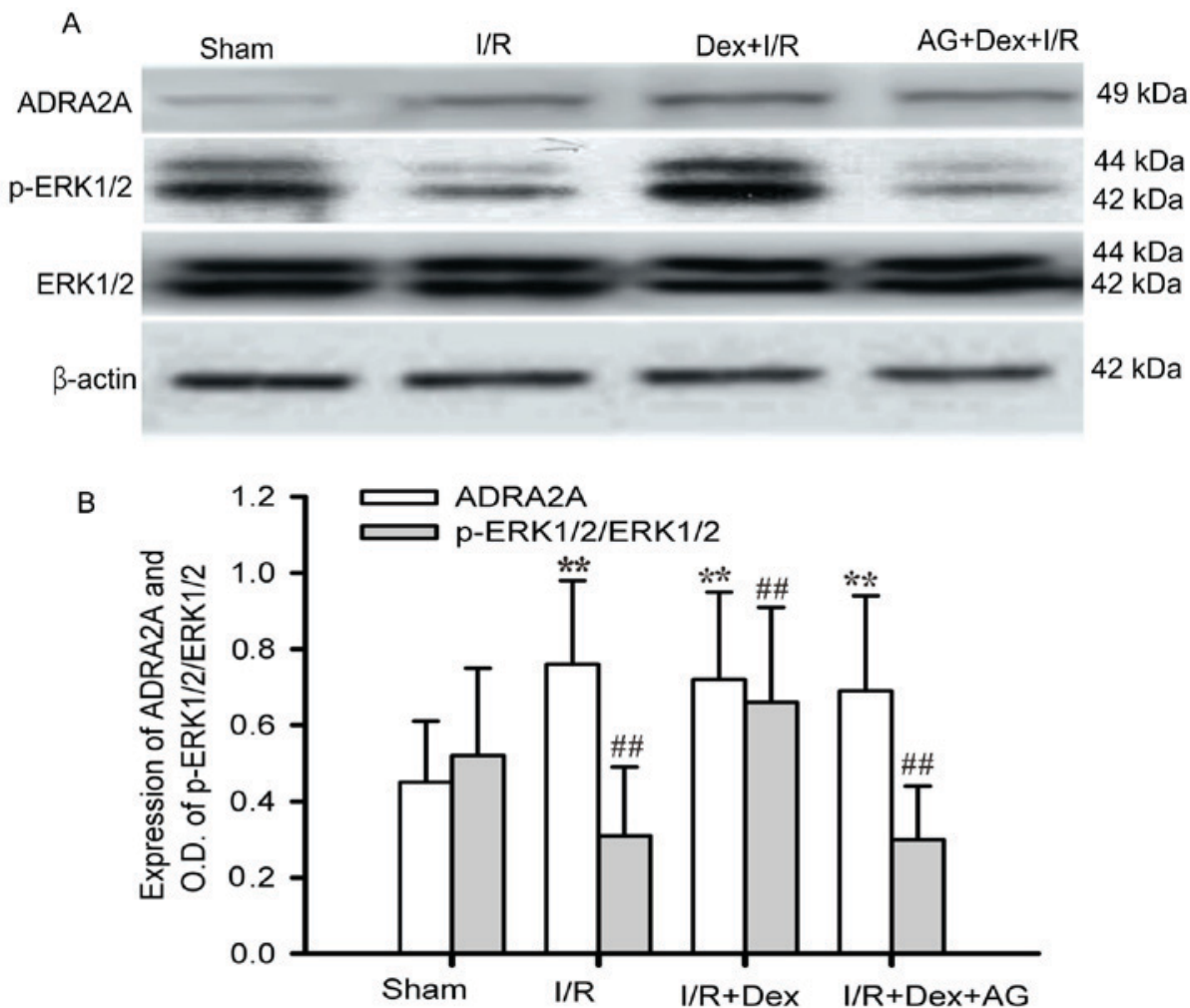

Figure 5. Effect of Dex pretreatment on ADRA2A and p-ERK1/2 expression in ischemic rat brain. (A) Expression of ADRA2A and ERK1/2 as detected by western blotting following different treatment conditions. (B) Quantification of western blotting data for ADRA2A and ERK1/2 expression following different treatment conditions. The relative optical density of p-ERK1/2/ERK1/2 was calculated, and the expression of ADRA2A and p-ERK1/2/ERK1/2 was normalized. Data are expressed as the mean \pm standard deviation; $n=6$ rats/group. ${ }^{* *} \mathrm{P}<0.01$ and ${ }^{\# \#} \mathrm{P}<0.01$ vs. control group. Dex, dexmedetomidine; ADRA2A, $\alpha 2 \mathrm{~A}$ adrenergic receptor; ERK, extracellular signal-regulated kinase; p-, phosphorylated.

$\mathrm{I} / \mathrm{R}$ injury regulates ADRA2A expression in astrocytes in ischemic brain diseases. In the present study, $500 \mathrm{ng} / \mathrm{ml}$ Dex pretreatment increased the expression of ADRA2A in the astrocytes. 
Dex has been demonstrated to evoke no ERK1/2 phosphorylation in cultured neurons but to activate phosphorylation of ERK $1 / 2$ in primary cultures of mouse astrocytes, and neurons could respond to astrocyte-conditioned medium with ERK1/2 phosphorylation (32). Dex may activate astrocytes through ADRA2A, and promote the release of different factors including GDNF or heparin-binding epidermal growth factor (EGF)-like growth factor (HB-EGF) to protect neurons following brain ischemia, potentially via different signaling pathways (10-12,32). Furthermore, Dex may induce GDNF release and increase the expression of $\mathrm{pERK} 1 / 2$ via mechanisms independent of ADRA2A activation. The I1-imidazoline receptors likely contribute to these effects (11). However, EGF receptor (EGFR) transactivation has been reported to lead to phosphorylation of ERK1/2 in astrocytes themselves and in adjacent neurons, because the astrocytes from the in vivo mature brain respond to a2-adrenoceptor stimulation (33). In the present study, Dex may have activated astrocytes through ADRA2A, dependent on p-ERK1/2 activation, indicating that other mechanisms enabling Dex to promote HB-EGF release through ADRA2A to protect neurons cannot be ruled out, and such need to be validated in further studies.

Dex concentrations of 25-500 $\mathrm{nM}$ can cause ERK1/2 phosphorylation in in-vitro cultured mouse astrocytes (32). The EGFR tyrosine kinase inhibitor AG1478, $\mathrm{Zn}^{2+}$-dependent metal protease inhibitor GM6001 and HB-EGF antagonist heparin can inhibit phosphorylation of ERK1/2 and EGFR (32). However, whether Dex pretreatment induces ERK1/2 phosphorylation remains unclear in in-vivo ischemic brain tissues with MCAO-induced focal cerebral I/R. In the present study, the results indicated that Dex increased ADRA2A expression in focal cerebral I/R brain tissues and mediated phosphorylation of ERK1/2.

The protective effects of Dex in cerebral ischemia were achieved presently with pre- and post-conditioning (drug administration prior to and following the ischemic episode), which is important in a clinical context, since its ability to protect brain tissue even after the onset of ischemia may increase its therapeutic potential as a neuroprotective agent $(34,35)$. The present results indicated that Dex decreased the inflammatory response of brain cells to ischemia, even when administrated $90 \mathrm{~min}$ after the H/R period, which indicated that it appeared to be a suitable and reasonable choice that Dex was administrated within 90 min after stoke.

In conclusion, a H/R cell model and focal cerebral ischemia model were successfully constructed in the present study in order to investigate the neuroprotective role and corresponding mechanism of Dex pretreatment in cerebral ischemia injury. Dex pretreatment appeared to increase ADRA2A and p-ERK $1 / 2$ expression in primary cultured astrocytes and rat ischemic brain tissues. Furthermore, the neuroprotective role of Dex pretreatment against cerebral ischemia injury seemed to occur via ADRA2A-mediated phosphorylation of ERK1/2. Since in vitro experiments cannot recapitulate all aspects of an in vivo model, the neuroprotective mechanism of Dex in astrocytes from cerebral ischemia tissues should further be identified through in vivo studies. Although many previous studies have used a sham group as a control to investigate the neuroprotective role and mechanism of Dex in cerebral ischemia injury in adult rats $(8,34,36,37)$, it may be more effective to have a sham group that also receives Dex. Elucidation of the neuroprotective mechanism of Dex in cerebral ischemia will better guide the treatment of ischemic disease, and targeting of astrocytes may be a novel treatment strategy for ischemic cerebral disease.

\section{Acknowledgements}

Not applicable.

\section{Funding}

The present study was supported by the National Natural Foundation of China (grant no. 81172467) and the Foundation of Health and family Planning Commission of Hubei Province (grant no. WJ2015MB153).

\section{Availability of data and materials}

All data generated or analyzed during the present study are included in this published article.

\section{Authors' contributions}

YS contributed to drafting the manuscript and the statistical analysis. XL constructed the in vivo model and experiments that followed. GL contributed to in vitro experiments. XP collected the data. MW designed the study and drafted and revised the manuscript. All authors have critically reviewed this manuscript and approved the final version to be published.

\section{Ethics approval and consent to participate}

The Ethics Committee for the Use of Experimental Animals at Tongji Medical College of Huazhong University of Science and Technology approved all animal experimental procedures.

\section{Patient consent for publication}

Not applicable.

\section{Competing interests}

The authors declare that they have no competing interests.

\section{References}

1. Ouyang YB, Xu L, Yue S, Liu S and Giffard RG: Neuroprotection by astrocytes in brain ischemia: Importance of microRNAs. Neurosci Lett 565: 53-58, 2014.

2. Tuttolomondo A, Di Sciacca R, Di Raimondo D, Arnao V, Renda C, Pinto A and Licata G: Neuron protection as a therapeutic target in cute ischemic stroke. Curr Top Med Chem 9: 1317-1334, 2009.

3. Barreto GE, Gonzalez J, Torres $Y$ and Morales L: Astrocytic-neuronal crosstalk: Implications for neuroprotection from brain injury. Neurosci Res 71: 107-113, 2011.

4. Takano T, Oberheim N, Cotrina ML and Nedergaard M: Astrocytes and ischemic injury. Stroke 40 (Suppl 3): S8-S12, 2009.

5. Lee TH, Kato H, Chen ST, Kogure K and Itoyama Y: Expression of nerve growth factor and trkA after transient focal cerebral ischemia in rats. Stroke 29: 1687-1697, 1998.

6. Afonso J and Reis F: Dexmedetomidine: Current role in anesthesia and intensive care. Rev Bras Anestesiol 62: 118-133, 2012. 
7. Kuhmonen J, Pokorný J, Miettinen R, Haapalinna A, Jolkkonen J, Riekkinen P Sr and Sivenius J: Neuroprotective effects of dexmedetomidine in the gerbil hippocampus after transient global ischemia. Anesthesiology 87: 371-377, 1997.

8. Zhu YM, Wang CC, Chen L, Qian LB, Ma LL, Yu J, Zhu MH, Wen CY, Yu LN and Yan M: Both PI3K/Akt and ERK1/2 pathways participate in the protection by dexmedetomidine against transient focal cerebral ischemia/reperfusion injury in rats. Brain Res 1494: 1-8, 2013.

9. Kose EA, Bakar B, Kasimcan O, Atilla P, Kilinc K, Muftuoglu S and Apan A: Effects of intracisternal and intravenous dexmedetomidine on ischemia-induced brain injury in rat: A comparative study. Turk Neurosurg 23: 208-217, 2013.

10. Degos V, Charpentier TL, Chhor V, Brissaud O, Lebon S, Schwendimann L, Bednareck N, Passemard S, Mantz J and Gressens P: Neuroprotective effects of dexmedetomidine against glutamate agonist-induced neuronal cell death are related to increased astrocyte brain-derived neurotrophic factor expression. Anesthesiology 118: 1123-1132, 2013

11. Dahmani S, Paris A, Jannier V, Hein L, Rouelle D, Scholz J, Gressens P and Mantz J: Dexmedetomidine increases hippocampal phosphorylated extracellular signal-regulated protein kinase 1 and 2 content by an alpha 2 -adrenoceptor-independent mechanism: Evidence for the involvement of imidazoline I1 receptors. Anesthesiology 108: 457-466, 2008

12. Yan M, Dai H, Ding T, Dai A, Zhang F, Yu L, Chen G and Chen Z: Effects of dexmedetomidine on the release of glial cell line-derived neurotrophic factor from rat astrocyte cells. Neurochem Int 58: 549-557, 2011.

13. Jana M, Jana A, Pal U and Pahan K: A simplified method for isolating highly purified neurons, oligodendrocytes, astrocytes, and microglia from the same human fetal brain tissue. Neurochem Res 32: 2015-2022, 2007.

14. Liu H,Davis JR, WuZL andFaez Abdelgawad A: Dexmedetomidine attenuates lipopolysaccharide induced MCP-1 expression in primary astrocyte. Biomed Res Int 2017: 6352159, 2017.

15. Chai L, Guo H, Li H, Wang S, Wang YL, Shi F, Hu LM, Liu Y and Adah D: Scutellarin and caffeic acid ester fraction, active components of Dengzhanxixin injection, upregulate neurotrophins synthesis and release in hypoxia/reoxygenation rat astrocytes. J Ethnopharmacol 150: 100-107, 2013.

16. Longa EZ, Weinstein PR, Carlson S and Cummins R: Reversible middle cerebral artery occlusion without craniectomy in rats. Stroke 20: 84-91, 1989 .

17. Levitzki A and Gazit A: Tyrosine kinase inhibition: An approach to drug development. Science 267: 1782-1788, 1995.

18. Alessandrini A, Namura S, Moskowitz MA and Bonventre JV: MEK1 protein kinase inhibition protects against damage resulting from focal cerebral ischemia. Proc Natl Acad Sci USA 96: 12866-12869, 1999.

19. Wang L, Lu YY, Zhou Q and Shui XL: Establishment and evaluation of a murine cerebral ischemia-reperfusion model. Hainan Med J 25: 2965-2969, 2014 (In Chinese).

20. Justicia $\mathrm{C}$ and Planas AM: Transforming growth factor-alpha acting at the epidermal growth factor receptor reduces infarct volume after permanent middle cerebral artery occlusion in rats. J Cereb Blood Flow Metab 19: 128-132, 1999.

21. Gu L, Li B, Yang X, Hu X, Huang X, Hertz L and Peng L: Depolarization-induced, glutamate receptor-mediated, and transactivation-dependent extracellular-signal regulated kinase phosphorylation in cultured cerebellar granule neurons. Neuroscience 147: 342-353, 2007.

22. Liu Z and Chopp M: Astrocytes, therapeutic targets for neuroprotection and neurorestoration in ischemic stroke. Prog Neurobiol 144: 103-120, 2016.
23. Barreto G, White RE, Ouyang Y, Xu L and Giffard RG: Astrocytes: Targets for neuroprotection in stroke. Cent Nerv Syst Agents Med Chem 11: 164-173, 2011.

24. Zhang S, Wu M, Peng C, Zhao G and Gu R: GFAP expression in injured astrocytes in rats. Exp Ther Med 14: 1905-1908, 2017.

25. Ma D, Hossain M, Rajakumaraswamy N, Arshad M, Sanders RD, Franks NP and Maze M: Dexmedetomidine produces its neuroprotective effect via the alpha 2A-adrenoceptor subtype. Eur J Pharmacol 502: 87-97, 2004.

26. Milner TA, Lee A, Aicher SA and Rosin DL: Hippocampal alpha2a-adrenergic receptors are located predominantly presynaptically but are also found postsynaptically and in selective astrocytes. J Comp Neurol 395: 310-327, 1998.

27. Hein L, Altman JD and Kobilka BK: Two functionally distinct alpha2-adrenergic receptors regulate sympathetic neurotransmission. Nature 402: 181-184, 1999.

28. Enkvist MO, Hämäläinen H, Jansson CC, Kukkonen JP, Hautala R, Courtney MJ and Akerman KE: Coupling of astroglial alpha 2-adrenoreceptors to second messenger pathways. J Neurochem 66: 2394-2401, 1996.

29. Hertz L, Lovatt D, Goldman SA and Nedergaard M: Adrenoceptors in brain: Cellular gene expression and effects on astrocytic metabolism and $[\mathrm{Ca}(2+)]$ i. Neurochem Int 57: 411-420, 2010.

30. Huang R and Hertz L: Receptor subtype and dose dependence of dexmedetomidine-induced accumulation of [14C]glutamine in astrocytes suggests glial involvement in its hypnotic-sedative and anesthetic-sparing effects. Brain Res 873: 297-301, 2000.

31. Jolkkonen J, Puurunen K, Koistinaho J, Kauppinen R, Haapalinna A, Nieminen L and Sivenius J: Neuroprotection by the alpha2-adrenoceptor agonist, dexmedetomidine, in rat focal cerebral ischemia. Eur J Pharmacol 372: 31-36, 1999.

32. Du T, Li B, Liu S, Zang P, Prevot V, Hertz L and Peng L: ERK phosphorylation in intact, adult brain by alpha(2)-adrenergic transactivation of EGF receptors. Neurochem Int 55: 593-600, 2009.

33. Peng L, Li B, Du T, Kong EK, Hu X, Zhang S, Shan X and Zhang M: Astrocytic transactivation by alpha2A-adrenergic and 5-HT2B serotonergic signaling. Neurochem Int 57: 421-431, 2010.

34. Wang SL, Duan L, Xia B, Liu Z, Wang Y and Wang GM: Dexmedetomidine preconditioning plays a neuroprotective role and suppresses TLR4/NF- $\kappa$ B pathways model of cerebral ischemia reperfusion. Biomed Pharmacother 93: 1337-1342, 2017.

35. Rodríguez-González R, Sobrino T, Veiga S, López P, Rodríguez-García J, del Río SV, Baluja A, Castillo J and Álvarez J. Neuroprotective effects of dexmedetomidine conditioning strategies: Evidences from an in vitro model of cerebral ischemia. Life Sci 144: 162-169, 2016.

36. Cheng J, Zhu P, Qin H, Li X, Yu H, Yu H and Peng X: Dexmedetomidine attenuates cerebral ischemia/reperfusion injury in neonatal rats by inhibiting TLR4 signaling. J Int Med Res 46: 2925-2932, 2018.

37. Yuan F, Fu H, Sun K, Wu S and Dong T: Effect of dexmedetomidine on cerebral ischemia-reperfusion rats by activating mitochondrial ATP-sensitive potassium channel. Metab Brain Dis 32: 539-546, 2017.

This work is licensed under a Creative Commons Attribution-NonCommercial-NoDerivatives 4.0 International (CC BY-NC-ND 4.0) License. 